\title{
Interview
}

\section{Managing cultural assets of Heritage Institutions - An interview with Rus Littleson of Media Equation}

\section{Rus Littleson}

has completed his BA (Psych) from University of Melbourne. He is the Managing Director, Media Equation Pty Ltd. He is responsible for the financial and strategic management of the Media Equation group including design, operations and the internet commerce franchise known as me-business. Media Equation Pty Ltd. (me) was founded in 1987 by Rus to take advantage of the perceived need in the market place to manage and automate the control of photographic images, by 'ingesting' digital images and then controlling the digital files. me operates both as a software developer and as a vendor of the products and services required by its customers from its Victoria, Australia Head Office. me products provide digital asset management (DAM) and digital rights management (DRM) capabilities.

\section{ABSTRACT Michael Moon interviews Rus Littleson of Media Equation Pty Ltd. on the key role of business processes to support digital asset management (DAM) systems for cultural institutions.}

Journal of Digital Asset Management (2009) 5, 196-215. doi:10.1057/dam.2009.16

MM: We are here with Media Equation, today. Rus - let us start with some background on your role at Media Equation, and a little bit of your history.

RL: I founded Media Equation as a company in 1986. In its early years, it was focused on marketing communications - in the 1990s. That quickly developed into the digital capture area. Then in the late 1990s, it started on the web-based road to DAM. We have been developing DAM systems since 1998.

Personally, I have been involved in the imaging area for quite some time. Before starting in DAM, we were specializing in digital capture. Before that, we were involved in marketing and communications. Understanding the processes involved in capturing images and using them in communications.

MM: Can you describe a little bit of your install base? What kinds of firms have deployed what, for Media Equation?

RL: We have just over 100 customers in Australia. Most of them are libraries, museums and galleries. We also have corporate
MM: Perhaps you can give us an overview of the state of DAM in those two markets.

$\mathbf{R L}$ : The corporate marketing sector is probably a little bit more developed because of the pressing commercial needs, to manage market intellectual property - brand management. In this sector, we have a number of installations that require very careful management of brand efforts.

After investing all their money in brands, clients want to make sure that their assets are used correctly. So we manage the correct usage of those assets via the system.

That is a relatively mature market, although the penetration is probably only $20-30$ per cent so far. Yet, there are many corporate entities that have not implemented systems.

The cultural and heritage system is probably a little bit further behind because of the volume and the cost. Comparing the sectors, in the corporate sector you might have 1000 or 2000 images. Even if a corporate client has a collection of archival images, it might not go over 10000 images. But in the cultural sector, you are dealing with 100000,200000 or possibly 1 million or even 5 million assets in the inventory under your control.

So it becomes a major cost, then, to digitize that collection. Raising money to digitize the collection has to come first. If you do not have 
the funds to digitize, then you do not have digital assets to manage.

A lot of cost will affect the cultural and heritage sector institutions, as they are struggling to raise the money. But it is becoming increasingly important. Governments are demanding access to images, and populations - the general public are demanding access. If you go into a cultural institution now and say, 'I'd like to look at such-and-such an item,' it costs money. It might cost the institution a few dollars to access this item.

In a library, it might cost the institution 10 dollars to get a book off a shelf, show it to someone and then put it back. Now of course, that is unsustainable in the long term, if you have the alternative available of showing an image of that item for next-to-nothing that is preferable, in terms of transactional cost.

MM: Right.

$\mathbf{R L}$ : The capital setup cost is obviously very high, to get everything online.

MM: Could you take us through some of the kinds of organizations that comprise the heritage section?

$\mathbf{R L}$ : There are both public and private entities in that sector. There are corporate sector clients that have historical images. There are private collections that are non-government, for example historical societies. There are indigenous groups with collections of archival assets.

There are regional interest groups - like groups of associations. Historical associations who follow a particular type of historical interest area with collections. There are a number of groups that would find it very difficult to raise funds for expensive systems.

At the other end of the spectrum, there are also organizations that can commit significant budgets. Large cultural-sector institutions - like museums and galleries - that are certainly well supported by federal or state governments and private donations.

MM: That could be at the federal or state or province level within a country?

$\mathbf{R L}$ : That is right.

MM: It also seems to me, Rus, that in a larger context, these cultural collections actually are part of the identity of a nation.

For example - Australia has a 'brand.' A story about what it means to be there - either to live, work or play or visit.
In that respect, a digital archive of the cultural then supports all kinds of stakeholders in 'Brand Australia.' To use, to amplify, to add new value to the national brand.

RL: Yes. It helps the rest of the world to understand the psyche of a nation. To understand the context of a nation. What is the history? Where have these people come from in terms of history? What has influenced their culture in the past?

As you know, the background of Australia since colonization is that the first fleets of ships that came out here held convicts. The State Library of NSW has digitized a number of first-fleet journals and made them available online through the system that we have implemented for them.

That is a fascinating story to read - the first-hand experience of the people who were on those ships, coming out to Australia - in shocking conditions.

There are journals from professionals that were on the boats who came out here to start a new life. Journals from the mariners, themselves.

So there is a range of different experiences that have been captured in handwritten journals, which have been digitized, transcribed, and made available online.

You get really good insights into the type of people who arrived here, and this can only help us understand better the nation we have today. MM: There is a parallel between the founding of modern Australia and the state of Georgia in the United States. The state of Georgia in the southeastern part of the USA was originally a penal colony.

$\mathbf{R L}$ : That is right!

MM: These visual collections - scanned images of journals - maps - diaries - newspaper clippings - paintings and drawings ... they are all, in a way, telling one aspect of the story of a people.

You could say that a cultural collection is really nothing more than a rich 'soup' of storytelling on the identity of a nation.

RL: Also to understand the threads of history that repeat themselves.

If you look at the concept of 'no taxation without representation,' and the similarity to what happened here in Australia with the Eureka Stockade, where a licensing fee was imposed on gold miners, with the Boston Tea 
Party. You start seeing parallels and similarities. The more of this information that is available online, the more we can understand humanity, really, as history unfolds.

MM: This also gets to a point that is quite relevant, today. More and more, we see government and private institutions digitizing their collections. They want to weave - into the fabric of education - the stories that these collections tell.

One of the primary constituent groups for these cultural collections is children in primary or secondary schools, developing cultural literacy.

Cultural literacy becomes essential to their citizenship and participation in the cultural activities, which directly relates to their efficiency and effectiveness as knowledge workers in a twenty-first century economy. $\mathbf{R L}$ : That is a major theme in the way that the Australian government is approaching education a theme of 'education for life.' An ongoing approach to lifelong learning.

I suppose it is one of the areas we pick up on in the way we promote our product and our service to corporate institutions. For example, with the state libraries, our proposed slogan for the program was 'from search to seduction.'

Everybody goes to a library or museum to look for something. With a library, most people are research-driven. You might go to a museum just to wander.

It is similar when you visit an online site for a library. Most people are actually looking for something. So the search is your primary aim.

MM: And it better look like Amazon, or I am out of here.

$\mathbf{R L}$ : That is right. So we want to make it a seductive process.

You go there looking for a certain thing, and you finish up immersed in the vast repository of knowledge that is there. Then you get seduced by everything else that is around you when you are going on a journey. You create your own story by joining things, too.

Like today - we have joined together the Eureka Stockade and the Boston Tea Party. Finding similarities or a nexus. That sort of storytelling and narratives, you can create individually.

MM: So you have surfaced now with another facet of storytelling in the cultural heritage domain. That is, increasingly, young adults and children today insist upon not just having things available at their desktops, laptops or mobile tops - but they insist that they be mashable. Such that I am co-creating my own narrative.

That puts certain demands on the technical infrastructure, to accommodate non-linear immersion in a vast body of assets. Can you speak to some of that?

RL: We have been through a period where content owners have tried to become contentaggregators. We have tried to aggregate disparate content into a single container, and present that as prepackaged entertainment.

I think the world has moved beyond that. As you say - people want to create their own stories, and customize them to their own names. Being able to go on a path or a journey within a particular repository, and find a storyline that tends to be linked with repositories that are held by other institutions as well. For example, between Australia and US institutions, being able to find links by easily going to the other institutions, and using very similar tools.

There has to be some sort of paradigm or homogeneity of how these tools operate. People expect that. As a platform model, you mentioned Amazon.com. People expect a platform to work like Google or Amazon.com does. So there are some powerful forces operating here. The challenge is to harness some of those paradigms into the cultural sector and build on them, so its easy for people to work their way through vast collections.

The State Library of New South Wales, for example, has more artwork than the Art Gallery of New South Wales - which is a major art repository in Australia. That is because of 'colonial art.' It is more of historical interest than artistic merit - granted. The information contained in that colonial art is enormous. At the moment - before it is digitized - you cannot find it. When it is digitized ...

MM: You still cannot find it.

RL: You will be able to have access to it. You still cannot find it. But Media Equation is making it available to the nation and to the world. We can then open up to possibilities of adding Wiki-type information to metadata. Being able to enlist experts who cannot see the image at the moment. Enlist those experts in 
the community who know about a particular area, to contribute valuable metadata to those images.

MM: Rus, I want to pick up on an idea that you introduced. I think it deserves a second look. The idea that as we start thinking about these cultural collections, we are really talking about how we surf and make sense of - and coalesce - multiple collections within that immersive discovery process of users.

Central to that ability to surf collections that span multiple owners, it requires a couple or three things, which I did like you to address.

First, it requires some integration. Some web services and/or data synchronization in terms of one system being able to read the other system. Right?

RL: Yes.

MM: The second thing is that it has to do with URL. A URL - without any description of it is pretty useless. Unless you click and go through it to see what is there.

What makes a file an asset is metadata. What makes any file an asset is metadata that - one describes what is in the file. As well as what you might want to search on. And, what sort of policies it might link to, in terms of rights and permissions.

You have just surfaced the idea of metadata or meta-tagging of URLs, where you are treating an URL as a new asset type within a repository.

Increasingly, that is where the value is going to be. The URL metadata becomes the 'airtraffic control system,' for at least how to identify that, 'At the other end of this URL is an asset that's described by XYZ parameters.' RL: Yes. We see the need for collaboration and sharing of items as becoming increasingly important on institution agendas. We have a couple of solutions that we have worked with institutions on, to develop or implement.

With a number of clients, we have an asset-sharing arrangement. One organization can propose to another organization that they share certain images. The other organization can accept all or part of the proposal. They can actually share digital assets.

The digital asset owner maintains control of the basic metadata, and the receiving institution cannot edit that metadata, but they can use the item and the asset in their system. That is something which is specifically developed between institutions that use our system.

Then you find that, well, that is great for people who are using the one system, but what happens when you have two different institutions using different systems? So of course there is the Open Archives Initiative (OAI) methodology of sharing images (see: http:// www.openarchives.org - the work of OAI has expanded to promote broad access to digital resources for eScholarship, eLearning and eScience). That has become very popular in Australia. Using the OAI protocol, we are able to share fixed or persistent URLs of images. To share selected metadata and an image of the asset with a federated search, more or less being able to go to one location and get images from a multitude of cultural institutions around Australia.

MM: The third item I wanted to also then have you speak to is this notion of 2.0 content, as 'asset metadata.'

As people start to create microblogs, or wiki-like annotations to an asset, that then becomes part of the metadata profile of the asset.

Increasingly, we want to search not just on the structured metadata, but on that unstructured folksonomy. Whether its a wiki, a blog, or some other kind of posted comment directly related to the asset and its meaning. We can then use that to better understand the context, and what we are looking at.

RL: Within the cultural and heritage sector, there are curators and custodians of cultural assets traditionally made fearful of opening up the metadata to the general public. Its more or less anathema to ...

MM: Library of science.

$\mathbf{R L}$ : However, if it is treated exactly for what it is, that is, an unverified contribution ... we steer away from saying that it performs as part of the metadata for the moment. We think just the concept of calling it 'metadata' is the basis of the trepidation in the cultural sector. MM: And yet, Rus, it is precisely metadata. It is data describing the essence of the asset. RL: And I think the cultural sector is coming around to it, to saying, 'Okay. The person who wrote that hasn't had 20 years' experience in classifying objects, but they have something to 
say.' So it goes into the metadata as exactly that. There is no dispute.

MM: Excuse me for jumping in here. But it seems to me, Rus, that you have this supplemental text that is essentially a narrative about the asset itself? It seems to be that a next-generation asset-repository for a cultural institution would want then to allow me to assign a provenance or a special consideration for wiki- or user-generated content that came from a certified curator - as opposed to a 14-year-old kid in his bedroom.

RL: That is right.

MM: So now we are looking at this usergenerated content, and then parsing it by its provenance and its authority.

RL: Yes. I think that is what it is. Being able to differentiate between the two different types of metadata has made more viable.

MM: That suggests that you are going to borrow some of the concepts prevalent in social network platforms, where if I have a profile -

a FaceBook page or a LinkedIn profile - and it carries some certification - suggesting my authority to speak on a matter - that then allows you to say, 'This comment comes from an expert.' That therefore is given certain consideration in terms of search criteria, as it relates to queries and so on.

$\mathbf{R L}$ : That is right.

MM: This gets to two deeper issues that I wanted you to speak to.

As we shift from what I call the '2.0 world' (user-generated content, attached to or associated with 1.0 content: HTML pages and videos), we find ourselves moving into a Web 3.0 context, characterized as publishing fairly standardized metadata.

In the context of financial information, we have a tsunami of change rolling through $\mathrm{XBRL}$ - the XML business reporting language, and through other sorts of industry initiatives, toward having an industry-wide standard metadata framework for content and information.

Now we are beginning to think about metadata as being another class of asset. Specifically, accommodating the social connections of metadata beyond the actual user permissions of the asset repository. Could you speak to this?

$\mathbf{R L}$ : I think one of the phrases you used earlier was the, 'next-generation,' of content. I think that phrase is useful. The 'next generation' of web users is going to expect the next-generation of web content to be more connected with social groups than the Web 2.0 type of metadata.

It is going to be increasingly important for cultural institutions to adopt Web 2.0 or Web 3.0 association of metadata with content. It will be important for cultural institutions to adopt that protocol, and to not be afraid of it.

So you take it for what it is. Declare it for what it is and manage that metadata. That is going to be important for cultural institutions, as well.

MM: The 3.0 concept of the Web is that it is not just metadata for content, but it is metadata for Web services of a service-oriented architecture. In a 3.0 world, I will have a browser with a widget. And the services connected to those widgets may come from multiple sources in the Cloud.

$\mathbf{R L}$ : Yes. That is right.

The specific example of that may be that when we demonstrated our product to some younger people, they wanted to use the Cooliris and $3 \mathrm{D}$ Wall to view the images. We are quite happy to present a traditional search-results page with thumbnails. But the younger the person and I am talking 10-13 years old - the more interested they are in viewing images in a 3D Wall. They would be able to download the Cooliris plug-in for Firefox, and apply it to images. That is the way they prefer to surf images.

MM: Right.

RL: It is not something that we had deliberately planned to promote as an add-on in what we do, but it is what younger people wanted to do. MM: Given that young people are such an important constituency for the cultural collections, it then has become for those cultural collections to make sure that their digital collections are remixable and viewable in multiple presentation theatres.

$\mathbf{R L}$ : That is right.

The adults, in general, do not want 3D Wall, and the younger generation does. There is a disparity between the demographics. We have got such a diverse range of demands and requirements. Everybody has to be able to choose their own presentation layer and also search methodology. 
Some people want to use Boolean search terminology to find things.

MM: I must say, Rus, I do not know a single person that prefers Boolean.

RL: A lot of people in libraries want to use it. MM: I understand. That is because they have library science degrees.

RL: Then you go outside the library, and people think that is terribly geeky. They do not want to have anything to do with it.

MM: What I want is a tag cloud.

RL: All of those users' viewpoints are valid, I think. Everyone has their own individual needs.

It is about personal choices. Your personal methodology. If a librarian wants to have Boolean search, give them Boolean search. If you want a tag cloud, you get the tag cloud. If you want a 3D Wall, you get the 3D Wall. Everyone has their own way of going about and doing things.

This is the increasingly diverse service demand that we have to meet.

MM: That puts a greater and greater emphasis on having a really well-developed metadata infrastructure.

It is more than just, 'Here are 10 pieces of data that describe an asset.' It will get into things like faceted taxonomies. Unique ways of organizing an entire collection or corpora of multimedia assets.

RL: Yes.

Having a mechanism for developing standards in those areas. In the short term, that is not going to be very easy to do. In the long term, it will. The community will develop standards over a period of time. But it is not going to happen immediately, within the Web 2.0 area.

It is going to be in question for quite some time. But obviously, with the structured metadata that exists, there are well-established, mature standards used by cultural institutions around the world.

We are going to have to allow for much unstructured, cloudy metadata for quite some time.

MM: That allows us to open yet another can of worms, as it is specifically related to cultural collections and digital archives. That is, user-generated content.

I daresay that most of the cultural artifacts of a country, an area and a people live in photo albums of citizens. Or shoeboxes filled with old Super-8 movies, video cassettes and so on.

One of the big things that we have seen develop here in North America in that particular sector - specifically at the level of county libraries and regional collections - is welcoming citizens to send documents that they want to contribute to the cultural commons.

There is usually some family pride in now being permanently ensconced in the cultural collection of your particular area.

RL: Yes.

MM: Could you speak to some of the technical and institutional issues around opening the collection - the digital archive - to citizens contributing material to the cultural collection. RL: Yes.

I think the idea of community contribution really clarifies the whole concept of community engagement and what can be expected from it. It sets expectations at a certain level with the people viewing the collection, for example Jewish Digital Narratives of the Judah L. Magnes Museum.

Everybody who goes to a collection of community contributions knows that it has not been digitized by the institution. The data that has been supplied with it has not been verified by the institution because of the sheer volume of data. It just would be impossible to do that.

Everybody goes into it with the clear understanding that it is a community project. The accuracy of the data there cannot be guaranteed.

MM: That would suggest that as a citizen, I have now gathered up some photos from my family album. I have scanned them on my little home scanner.

If I send them to you as attached files in an e-mail, one of the things I would like the repository to do - or at least the intake queue is to read the text in the e-mail as, 'descriptive, though unverified metadata.' So, part of a community of collections process would entail some way of having an e-mail become part of the metadata that a curator would then go through and utilize in a more structured way. RL: Yes.

We have implemented a DAM system at the Museum of Victoria. One of the projects that the museum has undertaken is a photo album, Melbourne's biggest photo family album. 
The general community has thousands and thousands of photographs being submitted. If you were describing one of your own family photographs, your metadata would have to be reasonably accurate.

But of course, there have to be some inaccuracies through faded memories. You might put something that is not quite right into the metadata. But it did give other people an opportunity to look at the data. So corrections can be made by other community members, as well.

It requires a thread rather than a moderator. Having a thread of information for an individual to assess the veracity of each contribution is probably better than having a moderator make an arbitrary decision on which one they believe. MM: That gets back to the idea of having a wiki-like or blog-like social media facility or service attached to the verification process.

RL: Yes.

That is technically a pretty simple process to have. It does not require a lot of disk space or bandwidth or anything. There is not a lot of technical impediment in doing that.

The biggest impediment is the political will to allow it.

MM: Yes. The correct mindset.

Rus, I am thinking that we might do a two-part interview. This might be a good time to wrap up this phase.

RL: Okay.

MM: Then in the next interview we will pick up on this thread. Where we then get into digital supply chains for content and media. Where we start to really explore some of the more structural and/or technical issues around workflow, collaboration, scheduling, rights management and so on.

$\mathbf{R L}$ : There is still quite a bit to cover. Is not there?

MM: Yes.

I think the piece that we have done so far is really brilliant. It really starts to unfold the larger case for DAM in cultural collections.

RL: Yes.

MM: It exposes that cultural collections become part of the identity of a nation or region. Two, making that collection available to children in school is imperative. Three, the cultural collection weaves a very rich story or narrative with many, many threads to the narrative. Each of which can be - to your point - a 'point of seduction.' That leads to this self-directed, immersive experience that is so natural and so much in demand by younger cohorts.

So in the course of these narratives that we want the repository to tell, it puts a premium on three or four technical features of the asset repository. One is that we have got really multidimensional metadata that allows for a variety of ways of engaging a large body of information.

Second, we have got the presentation layer abstracted to a point. If you want to simply do structured Boolean searches, you can. But if you want to have a 3D immersion marketplace, you can do that, as well.

Penultimately, user-generated content becomes more and more a major source of content and assets for the repository. This requires a different mediation process in terms of establishing the provenance and authority of contributors. Some of them may be certified, and some may not be.

This then gets into DAMs that become much more of a social networking platform. Specifically, by and for stakeholding members of the cultural collection. Some of them being highly credential curators, and some of them being curious kids that want to find out more about the place they live.

RL: Yes. The four aspects. That is really good.

There is one other aspect we could address post-interview, so we could wrap up.

That is the whole concept of online access for cultural institutions. Making it a community engagement exercise. It allows the cultural institutions to vastly expand their market reach and penetration in the community. Having physical space and physical exhibitions is limited.

If an organization has 5 million assets and their exhibition space can accommodate 5000 or 10,000 assets at any one time, it is going to take a lifetime to actually put all of those items on display in the one organization in the physical premises.

Having an online presence means that you open up the entire collection, eventually, to online access. That is something that a physical presence will never, ever achieve.

MM: I would like to use that as a coda for fundraising for these extended digital collections, 
which will have a lifetime many times longer than a physical lifetime.

So, let us come back soon and pick up on this thread of how digital expands the number of fundraising opportunities - as well as ongoing ways of engaging community into a collection that physical limitations of square meters of gallery space prohibit.

RL: Yes. Then of course once that is established in every cultural institution that has a sustainable platform for dealing with the 'now' generation which is not born digital, but digitized upon acquisition ....

As soon as an object arrives at an institution nowadays, it is photographed. That was not the case, before.

The volumes will start ballooning exponentially from now, and they have to get ready for it. They are not ready to cope, so they have to raise some money. It all comes back to the same point.

MM: It sure does.

Rus, for this next part of the interview, I would like to just restate several issues we have already covered, and then launch into the next chapter.

As we look at digital assets for the cultural heritage, one of the things that we discussed is how these digital assets become part of the brand - not only of the institution but also of the culture or society that it represents.

In case of the Library of New South Wales, that whole collection of assets becomes part of Brand Australia. As a function of that, the Cultural Heritage Institution is managing a regional brand. It has multiple stakeholders including other institutions, teachers, students and other members of that particular society.

When we start talking about younger users people that are still in grade school or high school or college - they have different media consumption habits - they want content to remix. Ultimately, they want to take those assets and use them in ways that perhaps no one really intended.

As these stakeholders in the institution begin to use these assets, oftentimes they want to post comments to them. So the notion of Web 2.0 becomes part of the overall metadata soup.

We discussed how some metadata is structured - derived from an authority list representing subject matter experts. Some metadata is just simply open-ended stuff that is part of the story. Not necessarily declared or identified as 'true' but somehow connected to the conversation and the underlying narrative of that particular asset.

RL: Yes.

MM: Finally, we talked about how cultural institutions must now start putting services in place to accommodate user-generated media. RL: It is a good summary. Starting with the brand and with the emerging trends in what the younger generation is demanding. That is going to shape what cultural institutions have to do in the future.

MM: Yes.

RL: We are dealing today with the commercial reality or the public need and demand on these institutions - what people expect from their institutions, in forming part of the brand.

MM: Then as we think about our current times with all the economic turbulence and uncertainty - this ultimately requires businesses as well as nonprofit institutions to rethink some of their core values, in terms of, 'Who are we? Why do we exist? How do we serve our stakeholders?' And if you are a commercial enterprise it is about, 'How do we stay funded? How do we make money?'

With the idea that this tsunami of change will continue to roll through the world, how do you envision or recommend that institutional stewards look at digital asset management as it relates to cultural heritage? How do they gracefully accommodate these necessary structural changes that result?

RL: I think one might have to look at getting a volume of material online, so they can remain front-and-center in peoples' consciousness.

In a contracting economy, where you have fewer people wanting to spend money on the gate price to any of the institutions, if you are charging an entry fee, you will see a drop in entrances.

So how do you stay in the consciousness of the people, where people will actually continue to support the institution as a valuable part of the community, warranting public funding?

Going online is one step in that direction. You can actually stay in the public consciousness via the content you put online.

I think in the case of the State Library of New South Wales, for example, we could have 
about 5 million artifacts. Where do you start, if you are going to digitize 5 million artifacts?

So far, they have digitized about 400,000 items of that immense collection. This tiny percentage - less than 10 per cent of what they have - has been digitized. How do they get through the rest of it?

A lot of institutions have duplicates of what other institutions have. Selecting what is unique and significant to the community and the culture of the place - the country or the region, is key to selecting what should be digitized and put online.

If it satisfies that criteria, then it goes to the top of the queue to be digitized and placed online. In the case of the State Library of New South Wales, the First Fleet journals from a variety of sailors and doctors and soldiers were made available online. They have been hugely successful and have really struck a chord in the community.

The web site that we did for the State Library of New South Wales was launched by a local actor that you may have heard of - Jack Thompson.

MM: Yes.

RL: Jack Thompson has taken an interest in genealogy. He has discovered through the research he has done that he actually is a descendant of a convict that came out in the First Fleet.

At the launch of the website, he was quite proud of that. He said he had not realized he was part of Australian Royalty. That is one of the ways that Australians look at it. If you have any link at all with the convict past, then you are really one of the privileged few to have that claim.

MM: There is a parallel, Rus, in American society - one of which refers to the first families of Virginia. Then there is the Yankee version of that, which would be the Mayflower Society, or the Daughters of the American Revolution.

RL: Yes.

MM: But it is quite funny that in societies where you had no royalty, there is always the 'We got there first' pride.

$\mathbf{R L}$ : That is right. Also because it is a dark past because of the convicts who stole a loaf of bread or something equally trivial, and were transported to Australia - it is a "small " $r$ " royalty,' and a claim to fame.
It is traditionally part of the AustralianAmerican culture, like the disrespect for authority that we claim to have.

MM: Yes.

RL: So Jack Thompson was quite proud to relate his discovery at the launch. I think everybody understood the significance of what was happening with taking all of these journals online. It is opening up the voices from the past, to enable you to see and experience that period.

Why do people claim this royalty as a privilege? To see where the modern nation's settlement has come from.

MM: While it goes to the particulars of the Library of New South Wales - and Australia, in particular, reveals a larger pattern, which relates to all cultural heritages.

One is that these collections really give substance and structure and detail to an underlying cultural narrative, to who we are as a people. Where we have come from, who we are today and who we are likely to be in the future.

In many respects, these assets become another mechanism of culture, by which to propagate from one generation to the next a certain set of social norms, values, beliefs and expectations.

RL: And knowledge. A DAM system is basically managing metadata. Metadata is information. But without a narrative, information is just that. It is information. With a narrative and connections and pathways, information becomes knowledge.

MM: That pulls forward another thread of DAM and cultural heritage. That is the knowledge in terms of connections and structures that bind a people. Ultimately, what cognitive scientists tell us - our brains are wired for stories.

The fastest way of training someone - one of the best practices for education - is to start with a story. One that is personal - or if not personal, deeply meaningful, and has many facets to emotional resonance.

RL: Yes.

MM: Back to your point with respect to what items we should curate, as digital objects. What were your two criteria? Important and significant?

RL: Unique and significant.

MM: What gives them their uniqueness - and to a lesser extent, their significance - is their connection to the cultural narrative that is the 
organizing principle or the framework for the institution itself.

RL: That is right.

MM: The other thing that you also highlighted here, Rus - because it was just too rich to let go by ... You had a well-known Australian actor that personally identified with the collection.

RL: Yes.

MM: Thereby giving the collection a face, a name, a personality and yet another reason to engage.

RL: Yes.

MM: So it seems that as cultural heritage organizations think about what to digitize and how to digitize it, another best-practice might entail then having two or three well-known people from popular culture become the 'face of the repository.'

So as some of us read gossip magazines, that same fascination with people and their back story will draw us into what is a never-ending story of these cultural artifacts.

RL: There is another way of doing this, as well, without drawing on the services of a current celebrity. A lot of institutions have material from famous people who have died.

MM: Right.

RL: They are custodians for the artifacts that belong to a particular artist or writer. There are very famous people who will create that interest, if people realize a certain institution has the material relating to a certain artist. They will create a big interest in that institution.

I have another example of that. The National Library of Australia - and the State Library of New South Wales both hold artifacts from the Australian author Patrick White. The collection held at the State Library of New South Wales for the author Patrick White includes letters that he wrote when he was 7 years old.

When he was 7 years old, he wrote letters to the fairies at the bottom of his garden. He wrote to them asking them to do something about the terrible flu that was going around.

When we were involved in the digitalization process for this project, which was one of the artifacts that we saw. Through the process we linked Patrick White's letter to the Spanish Flu, which devastated the world early in the last century.
At the time we were that digitalization process, the Avian Flu was sweeping the world. We discovered that the Spanish Flu was actually the first instance of the Avian Flu. So we had this incredible situation with metadata and the research revealing threads of connections.

People were excited by the fact that you could walk into and visit a library website, type in 'Australian Literature, Avian Flu,' and come up with a letter from 7-year-old Patrick White writing a letter to the fairies at the bottom of his garden.

People were excited to find out all the different celebrities and famous artists and creative people around the world who were caught up in this Spanish Flu. There were people like poet Guillaume Apollinaire and Egon Schiele, the artist.

A lot of famous people died as the result of the Spanish Flu, and here is a 7-year-old Australian writer who is going to be a worldfamous writer one day, trying to solve it himself at the age of 7 .

Incredible stories. The State Library of New South Wales is sitting on several floors of stories. Beneath its footprint - which takes up the equivalent of three or four city blocks, there are four floors below the ground and three floors above the ground. There are seven floors of artifacts - five million artifacts, and potentially millions of stories to be told.

Everybody is so excited that as the digitalization process proceeds, more and more of these stories come to light. So, in the future, you open up to the opportunity, via wiki, to have a world of researchers looking at material and offering contributions to what is there. Drawing pathways between different artists and creating the narratives that create knowledge for the community.

MM: Rus, it also seems to me that because you have such a large body of artifacts, the funding to then digitize and make that stuff available becomes a real creative challenge.

RL: Yes. You have to be very efficient. That draws on the e-commerce and monetization or commercialization of assets. So you can actually get some return on the investment.

It also requires a very efficient streamlined approach to how you were going to manage five million artifacts, for example. You need the business process management (BPM) systems in 
place. You need the e-commerce at the backend to create some revenue opportunities.

The BPM is a major aspect of justifying the request for funding to a board, for example. If you go to seek an endowment for a particular exercise - such as digitizing a collection - the question is, 'Is it sustainable? Are you going to come back next year and ask for more money? How is it going to work?'

It is important to create an efficient business process at the outset so that it is easy to increase the volume incrementally as the skills develop and experience accumulates.

There is the initial capital cost to set up BPM. But it will be recouped. That seems to suit the funding cycle of organizations. If you want to get the capital injection at the start, then you put your business case together and get the funding.

It is the recurrent funding that is very difficult to get. One of capital grants seems to be easier to get than recurrent funding because there is no end to recurrent funding. Whereas with capital, it is, 'Okay. Here's your one-off grant. Do a good job and we'll be happy. Put our sponsorship name on it and we'll be happy.'

If it is a government grant, it is 'Make sure that the constituency knows that when they come to vote at the next election that we did this for you.' So whatever the stakeholder is that put the money in - they have to be happy with the results of that one-off exercise.

Then the next year, who is going to fund the ongoing costs of running it? That is where being able to do it very efficiently and economically comes in. Also the e-commerce and monetization.

You can actually scale up and down, according to the revenue - very easily without actually having any break costs in your structure. You can do 50000 items a year or 100000 items a year. You will still have similar unit costs, once you have got your business process in place.

MM: Rus, this gets to a theme that we have developed in many of our interviews. Around the idea of a Center of Excellence. We would characterized the Center of Excellent, first and foremost, as an autonomous or semi-autonomous operating group within a larger enterprise or organization.
This center of excellence develops and employs best practices - generally from a larger group - association - community of practitioners. In this center of excellence, they use technology systemically to drive continuous ongoing process improvements, cost reduction, defect reduction and so on.

The center of excellence really is not just an operational capability, but it is a whole technical ecosystem that has a platform and ways of plugging into it.

This really sharply distinguishes two classes of enterprise DAM systems. One class of enterprise DAM systems has wonderful repositories. They have got rights management capability and rendering capability. Then there is a second class of enterprise DAM platform. They also have a really well-developed BPM capability.

So it is beyond just APIs and sockets. There is a real unique set of capabilities for developing complex processes. Could you speak to that? RL: Yes. That is a huge area of growth in the cultural sector.

On February 7th, New York Times published an article called, 'Digital Archivists Now In Demand.' A story that concentrates on UCLA and the process they are going through to digitize and manage collections.

They are requiring new types of skill sets within the organization, which blend digital knowledge with curatorial knowledge. Digital archivists are in demand. You have to have a digital ability as well as your historical cultural knowledge and expertise in managing archival collections.

It is going to be a huge growth area as DAM technology is rolled out, for people to be expert in both. A large part of the learning process will be the BPM methodologies that need to be implemented to make the whole process of having a DAM feasible.

You can have a million-dollar DAM installed and have no processes, and the material just trickles in, and the system will never get fully leveraged.

MM: It seems to me, Rus, that cultural institutions in particular have done some extraordinary work in codifying best practices for operations. I believe it comes from the Spectrum Partnership.

Could you explain a little bit about the Spectrum canon? And how that syncs up to an 
operational capability around cultural collections and digital archives?

RL: Spectrum standard comes from The Collections Trust Organization in the UK. Collections Trust is an organization that is empowered to bring best practices to not only the UK organizations, but also has an international approach. And it is part of the International Association of Museums, as well.

Spectrum provides a best practices approach to, covers management and documentation of a collection. This takes an interdisciplinary approach, blending technical and professional expertise with more general management skills in the overall management of a museum, including fund raising and service delivery.

So it covers all of the areas of operation in a museum. Starting with the pre-entry of artifacts. What are the things that the museum should do in terms of a legal environment and its own business policy requirements? To ensure that it can operate properly.

Then it looks at the processes around bringing an object or an artifact into the museum's collection, then, the management of it.

The Spectrum standard covers all of those. It is a very comprehensive standard that looks at all of the business process areas that a museum needs to cover.

MM: With all of the economic turbulence in the world now, it becomes really important for institutional leadership to really take their business practices to the next level.

RL: Absolutely. In fact, if we look at the whole concept of BPM, it is becoming embedded in the best practice standards of museums and cultural heritage organizations globally.

If you look at all of these best practices, they all flow into what I will call traditional process management concepts, which are explained by companies like the Process Factory.

They talk about taking strategic business opportunities, mandatory requirements, process improvement practices, things like Six Sigma normal business improvement proposals. It looks at how the business or entity needs to take all of those things - and run it through the board and management. What are the interdisciplinary requirements from the level of board member, director, partner, senior executive of an organization? And how they turn that to the efficient, effective and acceptable use of IT within their organization. To support repeatable, consistent business process executions - that comply with the rules, policies and procedures of the organization.

An industry example in the financial services industry here in Australia would include a major bank where Foreign Exchange traders got into problems. Internationally, one of the UK banks had a gentleman - one of their ForEx traders who got them involved in a huge scandal. A lot of the things that they were doing did not comply with the business's processes and policies. But there was no enforcement mechanism.

MM: As it relates to cultural heritage organizations, you could say that there are really two kinds of processes.

There are processes where basically you are manipulating - pushing around data and information. Whether that is transaction information about who bought what or what we need to order and when it needs to be here.

Then there are business processes that have large objects associated with them. For example, in manufacturing operations, you will have CAD drawings. You will have then the whole product lifecycle management around the certain of all your engineering documentation.

As it relates to digital asset management and cultural heritage organizations, for the most part we are talking about business processes that have very large files associated with them.

RL: And also, there are business processes that have to take into effect cultural and legal requirements in the area which they are operating. Be it the artifact - the provenance of the artifact - or the country in which they are operating.

For instance, in the United States, one has to look after and be aware of the cultural needs in Hawaii and not present images of deceased natives. It is a similar situation in Australia. One cannot - without prior written approval present images of a deceased Aboriginal person. In fact, when one is broadcasting a television show, one has to announce a disclaimer statement that this show includes information which may be pictures of indigenous people that are now deceased and that this may offend certain people. 
All of these things have to be embedded in the policies and processes, and ensure that anyone working to digitize and present a digital object to the world is aware of all of these limitations and can manage them - and work within the law. Otherwise, it exposes the institution to huge risks.

MM: Right.

RL: When I say, 'Risks,' normally these come down to having some large financial imposition as a result of transgressing.

MM: Yes.

This gets to an issue directly related to the BPM. Generally, within the larger workflow of how we ingest or intake new assets, and tag them appropriately. Then, manage multiparty rights.

Can you take us through some of the strengths and weaknesses of traditional DAM systems as they relate to managing multiparty rights?

RL: Yes.

Older DAM systems did not deal with rights very well. Because of the multitude of roles that could be ascribed in a moving image, there may well be over 20 parties involved who are stakeholders with rights to 'authorship,' or IP of or in that piece of work.

Being able to describe that adequately is quite complex. You need to have consistency of description of the roles, and the types of rights held by people. Being able to manage those and alter those, and describe - annotate - the rights is critical.

There is a vast array of information that has to be held in a database. Doing that efficiently requires a simple data-entry interface where it is easy to enter new information and enter all the different roles. Older versions of DAM did not do this very well, and it was quite a manual system. Newer versions can actually cover this, and it can become part of the rights metadata contained in the header file of the digital asset, because the standards have emerged, for example in terms of the XMP data that can be held in images describing the roles.

So rights information can 'travel with' the digital file, and be extracted by modern DAM systems, now. It is come a long way in the last few years because of the changes in standards. Being able to describe the rights adequately and manage them has become an important part, legally. The world is shifting to a more consistent global standard on rights management, and the expiry date on rights.

There is going to be more global, economic transfer of intellectual property. Having some standards uniform throughout the world will make that a lot easier.

For example, the 70 years from an artists' death rule - which is now becoming ubiquitous. Once that's established universally, everything will be a lot easier. In the major economies of the world, it is already there.

We now have a platform for managing rights internationally that makes the design of a DAM system a lot easier. Being able to ascertain the usage of an image or usage of an item to gather the project data and have the rights holders negotiate a price for the usage - it is a lot simpler now than it used to be.

MM: It seems to me, Rus, that from our previous points of discussion, many of these cultural institutions are part of a larger network be they federal, state or local networks of institutions.

We talked about how various cultural institutions will begin to syndicate portions of other institutions into their overall search and presentation experience. Take me through some of the challenges and solutions for managing rights in a federal distributed network of multiple institutions with multiple governance protocols.

RL: Well, the way it has been managed in Australia so far with the National Library which offers a federated search of state libraries is for the rights to be managed by the individual library. That is, the custodian of the item.

That is probably going to be the case for quite some time. You can go back to the individual institutions and say, 'I'd like to use this. I'd like to have this image for a certain purpose.'

But in terms of the search, the first step is - before you can federate an item - or offer a federated search for an item - in the national portal for searching state and regional collections - the state or regional collection has to make that item available for release, and has to declare it cleared for release. So it is able to be part of that federated search, because it is out of copyright - the artist has 
been deceased for 70 years, and the item can be used. Or it is come in to the public domain via some other means. Once that has been declared as cleared, an item can be in the federated search.

But going back, there could be some other items that are still within copyright, but the copyright has been expressed that it can be offered as part of an online collection. It might be a condition of the copyright.

But can it be used in a federated search? That is another question that should be sorted out by the curators at the local level. Can this be made available? What are the copyright conditions? Were they clear enough that they say, 'Yes. This can be viewable?'

There is a case going on at the moment where thumbnails are just below the copyright [rider] levels. So there is a dispute going on at the moment about the use of thumbnails - and whether they can be considered a breach of copyright.

MM: As I recall, that relates to specific adult content, and whether one adult website could steal thumbnails from somebody else, without paying them?

$\mathbf{R L}$ : Where there is money involved, let them sort out the legal precedent. That might impact on other areas of legitimate activity.

Generally speaking, in the cultural sector, it is not such a big issue. Eighty per cent of the material is out of copyright, and it is a vast collection of work. It is readily available for offering as a federated search.

It is a pretty easy process to go through and say, 'Yes. That falls into the out-of-copyright category. Let's work with that, first.'

So we are looking at 'first-tier' criteria, which were 'unique and significant' that one institution used, for example. That seems to be valid.

If you look at what you are going to take online first, it would be going through the filtration processes.

First of all, 'Is it unique?' Are we the only ones who have this item? Therefore, it makes more sense for us to be digitizing it first.

'Is it significant?' That is more of a curatorial/political/cultural decision. That is quite subjective.

The third one is, 'Is it out of copyright?' That is usually black-and-white. Sometimes it is a little bit grey, but it can usually be determined quite easily.

The three decision-making points for deciding whether an item is digitized - one of them has to be the copyright issue. Still, even if you go through that three filtration processes, you still finish up with a huge volume of work that can be digitized and displayed.

The copyright issue is something that a lot of bodies put up as a negative in the business case. Really, when you look at most cultural and historical, heritage institutions, they really do not have such a relatively big issue with the copyright as they think.

It is not absolutely small, probably, if you have got five million assets and one million of them are in copyright. Of course, one million copyright assets present a problem. But you have got four million left that you can actually deal with, without any legal problems.

MM: Rus, you did mentioned one particular idea here that I would like you to develop a little more closely.

In digitizing a group of assets, you have three different kinds of users.

There is a subject-matter expert - the frontline assessor. He says, 'Yes. This generally fits our criteria.'

Maybe that person then looks at the artifact and assesses whether it will require some retouching to make it more usable.

Then, in the next phase of the workflow, a curator is looking at this as a cultural artifact, and assessing its uniqueness, perhaps.

Finally, it is legal, determining whether the object is cleared for usage.

Did I get the sequence right?

RL: Well, there are two different sequences, one for 'born digital,' and another for analog data facts. Really, the accession information or accession decisions made by collections specialists will come first. 'Do we include this in the collection?' Then the preservation or conservation expert. The preservation/ conservation expert will assess the object for its stability. They will determine if any remedial actions need to be taken to enhance the preservation of the object, itself. And whether it can actually be moved safety to the digitizing area for digital capture.

Then the digital capture is made, and then the photographic expert will have a look at the 
digital quality, and whether or not something has to be done.

MM: So, the initial process is you are dealing with a proxy or low-resolution image?

$\mathbf{R L}$ : Or no image, as most artifacts have no image at all.

MM: Well, I would think that in terms of an online workflow, somebody would just take out a little video camera or a flatbed scanner and just do a quick-and-dirty scan. So we now have a portable, shareable file that we can throw out later if it, in fact, proves to be non-useful or redundant.

RL: Well I think that maybe, in the end, that might be a less-than-optimum solution. Most of the time that you are involved in the lifecycle of a digitization process is in the movement and management of the object, not in the actual digital capture.

You look at getting it off the shelf - moving it down the corridor to the photographic department - putting it onto a scanner or photographic device. Then putting it back on the shelf. Even if you have a transportable system that takes the actual digital capture to the object, most of your time is spent in the management of the workflow. Not the actual capture.

MM: I was thinking of almost like a little mobile phone with a 5-megapixel camera in it. Just to take a quick snap of it, as a visual placeholder.

RL: That may be done at the state level. The State Library of Victoria did that, as well. They used a video camera and captured tens of thousands of images. But it barely scratched the surface.

There were pretty much 450000 images, and that was going back a few years.

They had the advantage of having those quick-and-dirty thumbnails, so that there is a reference thumbnail. A lot of institutions have a significant collection of rough previews of the images.

But generally speaking, we recommend capturing some other workflows that exist in institutions for the purpose that you have described - a quick reference.

We think it is better to put the money into preservation capture. There is not a lot of difference between a preservation capture and a rough representation capture. But in any organization, we have found there are lots of processes going on. Where the exhibition department or the marketing department is actually taking snapshots of objects, using a digital camera, for their planning purposes.

Those digital objects - thousands and thousands of them - are being held on local hard drives from the marketing department or the exhibition design department. Those images are not going into the workflow.

Those images are actually valid images, as the recordings of the physical objects or the digital assets. They can be associated with the record if the workflow allows that to happen. They can be tagged exactly for what it is. That is, 'This is a shot that was taken by an exhibition curator, just for the purpose of planning and exhibition.' It does not have to claim to be a digital preservation image.

MM: That then really brings forward the idea that as we start looking at these preservation workflows and larger curator workflows, that we have various classes of users. They each want to look at an object or the digital representation of the object from a unique point of view.

Therefore, their user interface - and the associated metadata - must really reflect their subject-matter domain and the specific activities and tasks before them to complete. Can you take us through the thinking of separating metadata from presentation?

RL: Of course, it is part of this process that we are talking about. When the photographic expert is looking at an item, they are just looking at the photographic attributes of the digital capture. They are not necessarily looking at the rights information or the metadata or the provenance or the acquisition data or preservation details or anything. They are just looking at their own area.

So the various silos or departments of an organization can concentrate on their aspect their specialty areas.

The photographic department only has to look at the photographic quality. When it moves into the legal area, they just have to look at the legal aspects. Preservation only has to look at the preservation aspects of the artifact, and not the digital object.

When you have 5 million objects or millions of objects moving through a workflow over a 
period of years, you have got to track the process of where a particular item or batch of items is up to in the process.

That way, you can add metadata sequentially or out-of-sequence, according to the availability of resources. So if preservation gets ahead, they can work through the area. But the curators may not catch up with what the preservation people have done for quite some time. Or it could be vice-versa. The curators could get way ahead and they are waiting for preservation results to come back before they can actually proceed.

So workflow process management will help manage that, so different sequences and different processes can be conducted in parallel. Some of them have to be in parallel and some of them have to be sequential. There are dependencies.

The workflow has to manage all of those different variations and permutations of how something goes through from beginning to end, in the accession to public display.

Then once it is on public display, you have got the wiki opportunities for people to contribute their own metadata. Then there is the moderation of that. The workflow attached to moderating material or automating the moderation. They are all part of a global or enterprise-wide BPM solution that has to be implemented.

Once that solution is in place, you can grow your volumes very quickly.

MM: For the readers of this interview, not familiar with BPM as a category, could you take us through some of the fundamentals of BPM?

RL: For an example, with the Museum of Victoria solution that we have implemented: the workflow manages the accession of an item, and the different departments having a look at the item for preservation requirements, legal requirements and digital-enhancement requirements.

It can manage large volumes of images, and keep track of where everything is at a particular time.

The workflow engine should be able to manage specific pieces of metadata - and/or specific processes that are involved in preservation of an object. That will be less important for born-digital material in the future. However, the bulk of the material now is analog, and it needs to be managed, as well.
So BPM has to take that and everything into account. It is quite a complicated process. The idea of BPM is to make it simple.

BPM tools have to make the lives of curators, photographers and preservation experts easier - not more complicated.

We have a little example of a business process that we used when we talk to people about the simple process of asking someone if they would not mind getting you a cup of coffee, and how you would map that into a business process.

Once you put it on paper, it looks awfully complicated. You have to decide what type of coffee you are going to have. This is one of your decision points. Do you pay me now or do you pay me later? You are going down to the café.

The process that you finish up within the business process model looks complicated. But we know in practice that asking someone for a cup of coffee, and getting one, is quite simple. MM: It is a human being who shares a cultural context, against a background of obviousness as a philosopher once called it. It then becomes easy.

This is really one of the tough nuts that most people in BPM have to crack.

RL: That is right. Being human. Just going about your daily processes easily and simply. MM: So a critical factor then of effective BPM would be that first of all, you have to start with a set of good business practices. Second, you need a way of describing them in a uniform way.

Third, you need a platform by which to codify those best practices, using policies, routing and databases and other sorts of things.

Finally, you need to make sure that those business processes - once you have created them - can be changed fairly easily. So it is not like you are having to flip or set 1400 configurations of an SAP R/3 application. RL: Yes. I think generally there is a tendency to overcook business processes in implementation. There is a good example of that here recently, where a major airline in Australia implemented a BPM system for its spare parts. It spent millions of dollars implementing it.

Putatively it failed because the users had to answer far too many questions to get one bolt. 
In that scenario of course, everybody would want to circumvent the whole process and go straight to the store and just pick the bolt off the shelf. A BPM engine can become a white elephant and fall into disuse.

You have to make it very simple. Like the coffee example. If you actually had to fill out a form to get a cup of coffee, you would probably go off coffee.

I think you have to make it as simple as the reflexive process that you are talking about. It is easy.

You have a process with an inbox that says, 'What am I doing today?' 'Oh - I've got to capture that image, there.' You pick it up and you capture it and you move on to the next one. You give it a tick.

MM: So let us just develop this one last point, and then I want to shift on to funding strategies.

Specifically, the point that I would like to develop here is in terms of getting it as simple as possible - therefore elegant and immediately valuable. This requires that the business process modeling team not work from the top-down, but rather from the bottom-up.

RL: Yes. The executive officers that are doing the work have to design it.

MM: Right.

Specifically, the subject matter experts who are saying, 'Is this unique and important or significant?' It is really understanding what their job is. Almost as a software object, with inputs and outputs and so on.

Understanding how they work - and more specifically, how some automation would in fact enhance the way they work.

That takes an extraordinary amount of front-end innovation and collaboration with the actual stakeholders. Such that they automate their particular work cell or particular job functions as part of an overall platform as opposed to some custom APIs that I have kind of stitched together.

$\mathbf{R L}$ : That is right. You have to do it incrementally. Build it up so that you can establish a framework first, and you do not complete the process immediately, with the privileges and the mandatory fields and details like that. They can actually come a little bit later.

In the initial stages, all of those things get in the way of robust testing. If you can use systems in a simple form first, and then make it a little more complex as you go. The complexity should be below the surface. The surface layer should be really simple.

If you make it complex at the outset - if you have a theory of how it should work and you go and implement it - it may not work in practice. A lot of times, the change is simple, because the business process engines are geared toward change.

Nonetheless, it still takes a few days or hours to change every time you come across it if, 'That's not quite working. We have to change that.' If you constrain the system with privileges and multiple mandatory fields, it will be more difficult to change.

If you keep it simple to start with, and you do not have a lot of constraints built into the system, you can make those changes and remain agile in the early stages of the project. If you lock yourself into a particular very constrained way of working, because of the business processes that are perceived - rather than actual processes that are felt on the shop floor - as we experience the work processes and can alter them as you go, you will have a much better system within a few months.

If you spend a few months designing and implementing it, then you will often find out that it is not quite right.

MM: Sure.

RL: For quite some time. It will be a lot harder to fix.

So we have to go into funding.

MM: Yes. Let us talk about funding.

Specifically, the question is, 'What's been your experience in the cultural sector in terms of getting these initiatives funded?'

RL: Yes. I think Charles Rignall has got some specific information that he can share with you.

CR: The experience has been as broad as the number of customers we have dealt with.

There are some who have some very good visionaries. Again, it depends on the organization. When I say, 'The organization,' not just the people we are working with, but the people in the structure of the funding body.

It may be - for example - a state government institution that has to go to a committee, which then has to go to a minister of parliament - a local minister like a local government in the United States - to say, 'We need this much 
money out of your purse strings, from the state coffers. Please.'

Some organizations have been able to present information in a fairly simple way. And their management team or executive team has been able to say, 'Yes. This is all within budget of the operations.'

Others have presented it and found that the executive management just does not 'get it.'

MM: Right.

RL: 'What are we trying to do, here? What is this going to get?'

For example, we have got an institution that very clearly stated - that it has two key issues with what they are doing. One is the fact that they have very complex processes around what they do to get - for instance - an image request. For example answering a simple question: "We want an image to be able to be used in a promotional document for an exhibition we are staging. Do we have that image?'

Trying to answer such questions was unwieldy and complex. They found they did not have uniform or consistent processes across the different campuses and different departments.

So they captured what should be the process. Once they designed the process, they needed to enforce this across all of the different campuses that form this institution. Think of it as something like the Smithsonian - with different departments and different campuses.

They developed a policy-and-procedure manual related to how they were going to manage images. How they were going to request images. How they were going to name things.

When they did all of that work, they realized that they needed to implement a DAM system. They found that everything they did showed they needed DAM technology to underpin and integrate with their business processes.

They worked with us and we helped them develop their business case doing the normal things: 'What's it going to cost? What's it going to do? How much time are we spending now?' Before-and-after and so on.

We helped them write a financial business case, which they were able to get approved, and then go to tender - and finally, they installed our product.
Another institution had a project and had already received significant government funding and private-investment funding to say, 'Yes. We are going to digitize our assets because we need to preserve them. We have got various things like manuscripts, documents, paintings, et cetera, that are aging. They will not last. We want to preserve them.'

Digitization was determined to be the only way forward. This was a library.

They also wanted to be more relevant to the public and its constituents. This library had a collection of artifacts and books - their purpose or charter is to expose the collection to the public for research and sharing of information. Not just the public, but other institutions, universities and schools.

They also realized that as one of the other side effects, there is a 'greening' to be had. Because one can reduce the carbon footprint of the research effort required, if you can make available information which can be browsed via the web - rather than people hopping on a bus or a train or into a car and driving to the institution and spending hours in the institution burning up power and various other things within the gallery, to get all of the information they need. Plus, to make multiple photocopies and get rights of approval to do this, that and the other things that one needs to have to get data out of the books, manuscripts and so on, that you are referencing.

So they had the digitization project in place. We worked with them and won a tender to complete the project. To take the process of digitization with our BPM system - to manage the digitization process and the ingestion into our DAM. And then to manage the distribution and access via the web.

I will read what, David Drinkwater, Project Manager, State Library of NSW said in describing the outcomes: The 'project has delivered the State Library of NSW a sustainable and extensible software platform for management of web content, digital assets, business processes and archival collections.' As a result the executives of the library were able to go to their benefactors and the State and get funding for a further US\$20 million to complete the digitization of the first five million artifacts.

It is one of those things. You take an idea and a concept, and a lot of people have 
difficulties imagining or visualizing it. Suddenly when it is made real, they see the benefits. It is like the Aladdin's Cave effect. Wow! What is in here?

MM: Yes.

RL: It is also given them some more creative opportunities for funding. They created what they called 'Journeys of Discovery,' which are journeys through History. So, there are various subject/theme matters. They put together objects for the public to get an appreciation of the history of that phase or area of Australia's history. This further emphasizes the 'search to seduction' theme.

There was - for example - the era of Governor Macquarie - and there was a major bank in Australia called 'Macquarie Bank.' The Library was able to get Macquarie Bank to provide sponsorship for that journey.

They were able to get some creative marketing to go to corporations to make significant donations against the specific journeys. So, a Horse Stud did something about racing in Australia.

Great tie-ins. It is given the institution another avenue to attract donations. And more importantly, to recognize those people who are making those significant donations to the institutions.

MM: Right.

$\mathbf{R L}$ : It is a real win--win.

MM: It makes complete sense. I really like the idea of the 'journeys.' The various journeys, using digital assets as the bricks.

There are all of these various journeys, and those then become brandable events or brought to you by XYZ Sponsor.

RL: Correct.

MM: Are there any other formats for inducing sponsorship? Specifically with respect to ingesting and preserving new classes of assets? And/or partitioning them in special collections 'brought to you by ... ?'

RL: Yes. There are. This, again, depends on the capabilities of the digital asset management system. But it is one of the capabilities that we have. You can create mini collections or albums.

People can see mini collections that are exposed. You can brand those mini collections as, 'Brought to you by so-and-so.'

Another way of doing it - again - comes down to the institution. How it wants to do its marketing and branding, and what its policies and procedures will allow.

We have done a lot of work mostly with institutions which are state- or federal-government owned. One institution approached us to help them with their fundraising project. We created some collateral for them - which was an example or a demonstration website. Saying, 'This is the library of the future.'

The name of the library fundraising project was slv21 and we created a website called slv21com. We also created an interactive CD-ROM, which repeated the experience from the web.

This covered some of the key areas of digitization, such as taking manuscripts and making them page-turning experiences. So that you presented the capability of a book or a manuscript, which the viewer - the reader on the web - could turn the pages by hand; by using the mouse to create that interaction.

Again, it all adds to the theme of not just presenting information, but displayingdisplaying it in a seductive way, to seduce the browser to want to stay and explore more.

It was their Library of the 21st Century, and we helped estimate a number - with them - to a dollar value of about $\$ 21$ million to achieve the first phase of their project.

The result of this was they were able to go out and get funding to the value of $\$ 21$ million. They were able to explain to potential donors what they would deliver.

MM: Right.

It is almost like a real estate developer putting in his master plan for a golf course.

RL: Yes.

MM: And then to say, "Well, on what course would you like to have your fabulous new dream house?'

RL: Yes. Exactly.

Or a developer saying, 'All right. I'm building a 36-hole golf complex. I'm going to be naming different holes after different people,' instead of how the Masters' has the Magnolia Hole. This is going to be the Century 21 Real Estate hole. 'You've got naming rights in perpetuity, there. Will you help fund the development?'

MM: That also includes all of the related services associated with the acquisition, digitization and clearance and so on?

RL: Exactly. But it opens new avenues for benefactors to be recognized. We really should 
not minimize that. A lot of institutions just generically recognize significant donors as gold donors or however they choose the different levels. Based on the amounts of money people promote.

MM: Right.

$\mathbf{R L}$ : There is very little opportunity for donors to say, 'I really want to be involved in projects that are good for the greening of the earth,' or, 'I want my money to go toward your collection of books for the library that deal with this subject matter.'

MM: These are really themed and/or valuedriven endowments.

RL: Correct.

And the digital technology and digital asset management really allows that benefactor or endowment organization to sponsor very specific things - consistent with - oftentimes the giving organization's mission. As well as the benefactor's personal interest or desire to see propagated into perpetuity.

RL: Exactly.

So the legacy becomes very personalized for the benefactor.

MM: So as a living trust - as a financial institution for carrying and maintaining the integrity of an estate - we are now talking about a digital collection or a digital heritage that has its own life and carrying forward. Maintaining its integrity as a collection.
RL: Correct.

MM: Wonderful.

Before we wrap this up, are there any final comments that you would like to leave us with?

RL: I think there are three key comments I would like to make. As far as we have come with DAM - and it might be a cliché - we have really only just scratched the surface.

MM: Yes.

RL: But for everything that we are doing, I think the overarching concepts are that whatever institution or organizational company we deal with, those institutions are only the sum of their business processes. So, DAM is the technology we focus on. The business processes supporting the digital assets and the DAM system are the real key functions of that organization.

I think it is important for anyone looking at any technology to look at the appropriate processes that support the way that technology is on-boarded to their organization. That is where I think we have been most successful in helping our customers become successful.

MM: Absolutely.

I want to thank you again for spending the time with us here, today. Great success in carrying forward with cultural institutions around the world.

RL: Thank you very much. Much appreciate your time, Michael. 